CERN-SL/95-82

18 September 1995

\title{
VERTICAL BUNCH-SIZE MEASUREMENT AT LEP USING VERY FAST X-RAY DETECTORS
}

\author{
D. Cocq, A. Manarin, E. Rossa and J. Spanggaard \\ CERN, 1211 Geneva 23, Switzerland
}

\begin{abstract}
Very fast X-ray detectors have been developed for beam diagnostics in the LEP machine (Large Electron-Positron Collider). Picosecond CdTe photoconductors allow the measurement of the vertical dimensions of electron and positron bunches (length $50 \mathrm{ps}$ and vertical size 300 microns) during a single beam passage. Intense synchrotron X-rays emitted by the LEP beams are measured each turn by two sets of detectors.

The transverse profile detector and its associated electronics are described in detail. A pulsed bias is used to gate the detector for a period of time as short as $10 \mathrm{~ns}$. The profile is then digitized in $10 \mu \mathrm{s}$ and saved in memory. Typical results obtained in real time with LEP beams are used to illustrate the performance of the detector. The linear pulse bias provides a new way of simultaneously making single-shot Xray measurements of the longitudinal and vertical bunch centre of gravity, and the vertical profile.
\end{abstract}

Submitted to the 9th International Workshop on Room Temperature Semiconductor X and Gamma Ray Detectors Associated Electronics and Applications, Grenoble, France, 18-22 September 1995 
Very fast X-ray detectors have been developed for beam diagnostics in the LEP (Large Electron Positron Collider). With each passage of the same bunch of particles, the vertical and longitudinal dimensions are measured in a single shot. LEP positrons and electrons yield a lot of synchrotron X-rays in the bending magnets. Since the emitted photon pulse has the same shape as the corresponding bunch of particles, its analysis will provide information relevant to the beam itself.

The first design [?]-[?] of the detector was made for the vertical dimension (sigma) in the range of a millimetre, and the X-ray bunch length was about $30 \mathrm{~mm}$ (sigma of $90 \mathrm{ps}$ duration). The time interval between two successive bunches was $22 \mu \mathrm{s}$. With the improvement of the operation of the LEP machine there are now bunch trains of particles circulating in the ring, with only $237 \mathrm{~ns}$ between two bunches and all spatial dimensions smaller. The X-ray detector has been adapted by increasing the speed of the acquisition system; moreover, a very precise timing pulse-bias allows a selection of bunches in the train. This linear pulse bias provides a new way of simultaneously making single-shot Xray measurements of the longitudinal and vertical bunch centre of gravity, and the vertical profile.

The present paper describes the vertical profile detector, while the bunch length measurement is developed elsewhere [?]. The electronic circuits are the same for both detectors and are analysed here before giving some examples of the results obtained with the beam.

\section{DETECTORS}

The detectors, which use CdTe photoconductors, have been described in previous papers [?]-[?]. Here, we simply recall that a detector array has 64 equally spaced 'channels' with a pitch of $100 \mu \mathrm{m}$ (see Fig. 1). A thin layer of CdTe $(4 \mu \mathrm{m})$ is deposited on a ceramic support $127 \mu \mathrm{m}$ thick, using the metal-organic chemical vapour technique.

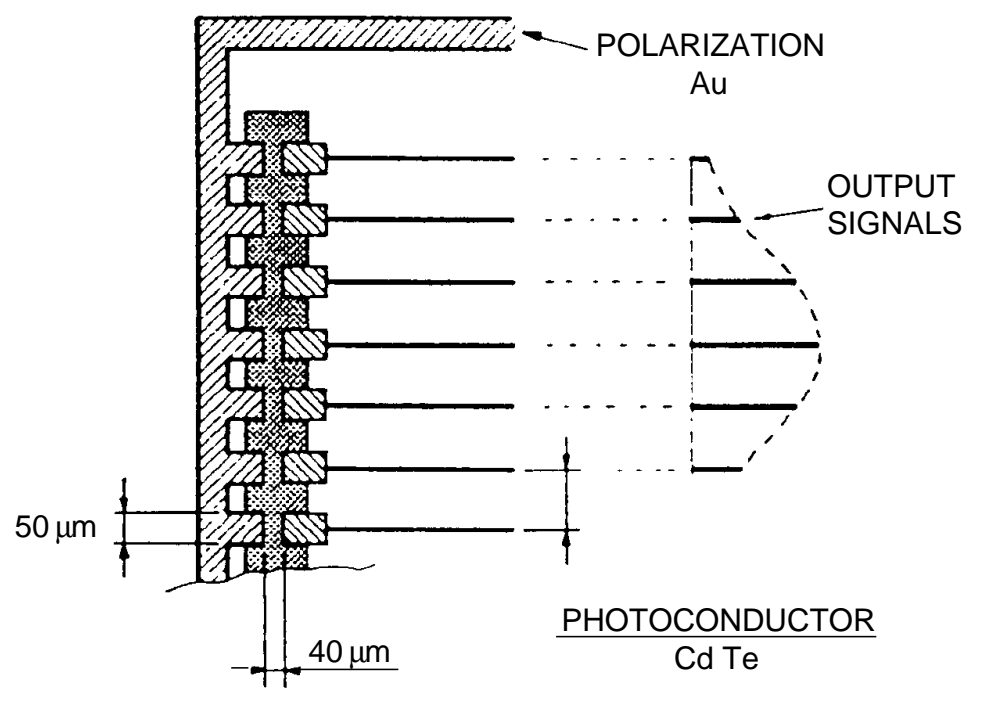

Figure 1: Layout of the detector for vertical profile.

Under the impact of X-rays, the conductance of CdTe changes and the gap resistance decreases as a linear function of the photon flux-generating currents in the different lines directly giving the vertical profile. 
During LEP operation these detectors can be permanently used in parasitic mode, although they are exposed to doses of X-ray $(10-100 \mathrm{keV})$ irradiation of up to $10^{12} \mathrm{~Gy}$ per year. The polycrystalline CdTe grown by metal-organic chemical deposition is very radiation resistant. During the feasibility tests, the detectors were exposed to intense Xray radiation $\left(6 \mathrm{~W}\right.$ per $\left.\mathrm{mm}^{2}\right)$ for a month. This resulted in the deposition of a carbon layer on the photoconductors due to residual gases, with carbon producing a shunt across the photoconductors and increasing the dark current. New detectors installed in the machine have been fabricated with a protective $\mathrm{SiO}_{2}$ isolating layer.

Detectors made elsewhere [?] show a single crystalline CdTe layers grown by molecular beam epitaxy (MBE). There is no publication of the effect of very high intensity $\mathrm{X}$-rays $(>100 \mathrm{keV})$ on this single crystal. It is probably more sensitive to the default created by the beam than either a polycrystalline or amorphous material.

\section{MECHANICS}

Movable mechanics support the detector mounted on a water-cooled aluminium block, allowing it to be displaced vertically within a range of $20 \mathrm{~mm}$ and rotated $45^{\circ}$. The movement allows for a proper alignment of the detector with the incident synchrotron Xray and its retraction from irradiation when no data is acquired. The detector is electrically isolated from its support and is contained in a vacuum tank $\left(10^{-6}\right.$ Torr $)$.

\section{FRONT-END ELECTRONICS}

Radiation-resistant cable has been designed to connect the detector to the frontend electronics through the vacuum port. This long, flat cable is made by industry to CERN specifications. There are $64 \mathrm{Kapton}-i n s u l a t e d$ strip-lines carrying signals and two polarization lines. The printed board circuit keeps the width of this cable to $20 \mathrm{~mm}$ despite a length of $1.8 \mathrm{~m}$.

All front-end electronics close to the detector have been built with the prerequisite for using the digitizing and storage system already developed for other equipment. Since the detectors are situated in the LEP machine's accessible pit $300 \mathrm{~m}$ away from the digital processing, the following analog transmission was used.

One set of detectors is made up of a 64-channel vertical detector plus 16 channels of longitudinal autocorrelator [?]-[?]. In order to reduce the cost of transmission cable for all these analog signals a time multiplexing system was developed. Since the input flash ADC was able to take data spaced by about $1 \mu \mathrm{s}$, it was decided to time-multiplex 16 signals on the same cable in less than $22 \mu$ s (interval between LEP bunches at the early start-up).

There was no electronic module available on the market for integrating picosecond pulses or for storing and timing multiplex 16 output signals, each of $1 \mu \mathrm{s}$. Therefore, a VME module with 16 input channels was developed at CERN. In this module the charges are integrated (see Fig. 2) by a LeCroy HQV hybrid amplifier. The risetime of this very low noise amplifier is only $10 \mathrm{~ns}$. Compared to the $50 \mathrm{ps}$ input pulses it would seem not fast enough, but the input cable acts as a capacitative memory buffer. When the input pulse returns after the first reflection on the input of the amplifier the photoconductor is already off. This impedance is above a number of $\mathrm{M} \Omega$ and the pulse comes back again to the input of the amplifier until the charges are fully transferred in the integrator. The charges are then stored and time multiplexed by one other hybrid module (MIC) also from the firm LeCroy. 


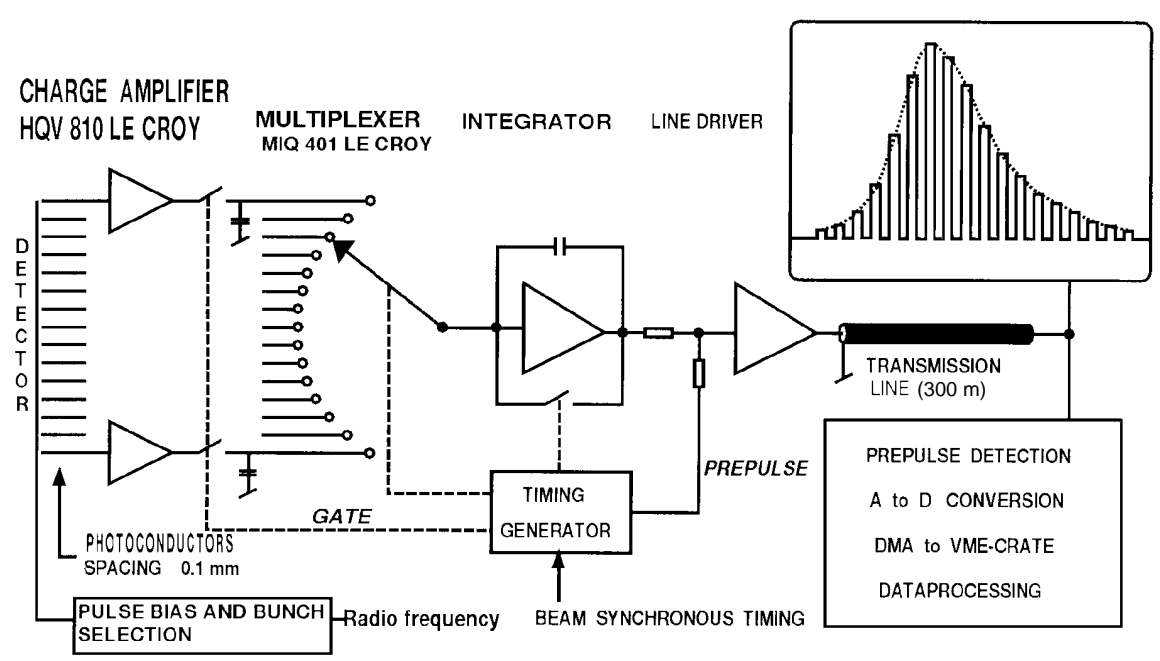

Figure 2: Synoptic diagram of the detector and front-end electronics.

All the functions shown by the synoptic diagram of Fig. 2 are realized by a very fast ECL circuit in this hybrid module. Finally, one other integrator and the line driver sequentially convert the charges stored in the MIQ, in pulses in which the amplitude is proportional to the stored charges. A prepulse is mixed at the beginning of each piece of data and is afterwards useful for precisely synchronizing the digitization.

In this way, 16 channels of the vertical profile can be sent as a $16 \mu$ s-long train of pulses containing trigger information for the flash ADC $300 \mathrm{~m}$ away. The electronics are the same for the other detector developed to monitor the autocorrelation of the bunchlength profile. The front-end module has been designed with two outputs, with the low sensitivity one being attenuated by a factor of eight, in order to increase the useful dynamic range of the 8-bit flash ADC.

The high sensitivity output gives $4 \mathrm{~V}$ amplitude for a charge of $50^{-15} \mathrm{C}$ received in a pulse where the pulse width can be only some picoseconds.

\section{$5 \quad$ ACQUISITION SYSTEM}

Figure 3 is the synoptic of the entire acquisition system.

Through these electronics the signals received by each channel of the detector are digitized and recorded in an acquisition memory, turn by turn and bunch by bunch. It is possible to program the electronics to record only selected bunches. The capacity of the acquisition memory is such that 224 turns of eight bunches, or 1600 turns of one selected bunch, can be stored. Once the data are stored, a software process scans the memory and applies calibration coefficients (offset and gain) to reconstruct the beam profiles for each recorded bunch and turn. These profiles are stored in a binary table and transferred to a central workstation for permanent storage and analysis.

The same VME crate containing the electronics also contains a 68030 -based CPU, running OS9, such that all hardware parameters are under the control of multiprocessor software. A brief summary of the parameters:

- displacement of the detectors in two orthogonal directions;

- change of the polarization voltage;

- performance of bunch selection;

- selection of the number of turns and the interval between turns; 
- selection of a trigger for starting the acquisition (machine event - such as injection prepulse or external timing).

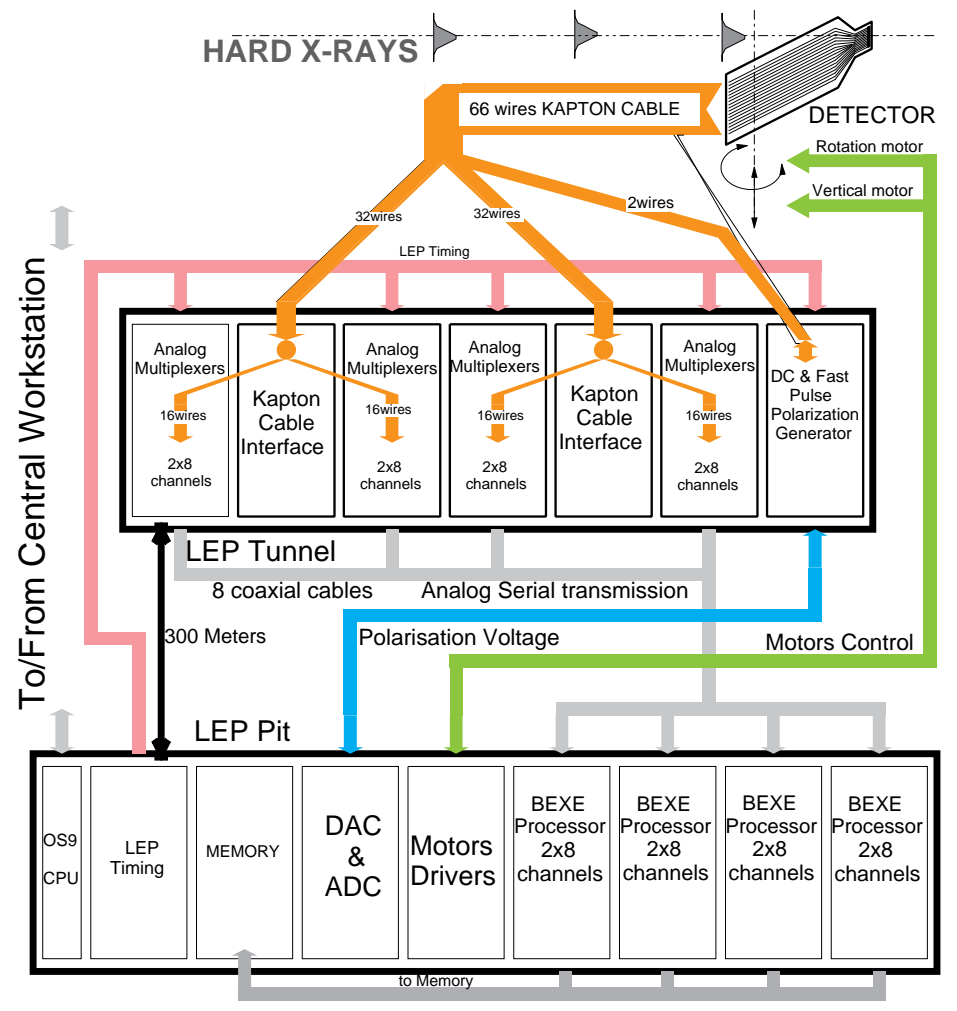

Figure 3: Schematic of the acquisition system.

One interesting feature of this software control is the powerful calibration of the entire system with the real X-ray beam. When the machine is stable the detector moves in small steps slowly through the beam and each channel records a very large number of turns. By comparing the different values recorded by each channel a calibration factor can be determined. The offset of each channel is measured directly without an X-ray beam on the photoconductors.

Data are subsequently stored on an HP workstation and can be analysed in different ways [?]. FFT's applied to vertical bunch position and sizes have revealed particular oscillation frequencies.

\section{NEW DEVELOPMENTS}

\subsection{Pulse bias operation}

The new way to operate the LEP machine using bunch trains, where the time between successive bunches is only $237 \mathrm{~ns}$, was not foreseen when the front-end electronics were first developed. The current generated in a photoconductor is proportional to the product of the flux of photons multiplied by the voltage across the gap. Thus we have built a new, fast photoconductor pulse-bias in order to be able to select individual bunches in the train.

The problem that had to be solved quickly and without spending too much money was not a simple one. The bias line on the detector itself and the bias strip line in the Kapton cable were not designed to carry nanosecond pulses. Finally, it was decided to 
send a triangular pulse bias on the photoconductors at the maximum rise time $(5 \mathrm{~ns})$ allowed by the $2 \mathrm{~m}$ cable.

A careful analysis of this type of pulse has shown two unexpected advantages. First, the parasitic voltage induced by the bias line in the adjacent channel (1 and 64) is bipolar and fast enough so that negligible charges are integrated in those channels.

Furthermore, the total intensity recorded by the entire detector could give other interesting information about the position of the longitudinal centre of gravity of the Xray pulse. Formally, this is a precise turn-to-turn measurement of the fluctuation of the phase of the particle bunches, relative to the radio frequency signal when it is used to trigger the generator. This is demonstrated below.

A very precise timing module mentioned in Section 6.3 was used to synchronize the pulse bias generator and gate the desired bunch in the train.

\subsection{Longitudinal centre of gravity of X-ray pulses}

When the beam circulates in the LEP ring the number of particles per bunch is constant from turn to turn. Thus the total X-ray flux emitted per bunch in the bending magnet is also constant from turn to turn, and is proportional to the intensity of each bunch.

The total current $\mathrm{i}(\mathrm{t})$, created by $\mathrm{X}$-rays in the 64 channels, can be written as:

$$
\mathrm{i}(\mathrm{t})=\mathrm{ku}(\mathrm{t}) \Phi(\mathrm{t})
$$

where $\mathrm{k}$ is a coefficient, $\mathrm{u}(\mathrm{t})$ is the bias voltage and $\Phi(\mathrm{t})$ is the time distribution of the $\mathrm{X}$-ray pulse (which is equivalent to the longitudinal distribution of the particles in the electron or positron bunches).

If we use a linear rise pulse-bias then the voltage across the photoconductor can be written as:

$$
\mathrm{u}(\mathrm{t})=\mathrm{at}
$$

where $a$ is the slope expressed in volts per picosecond and $t$ is the time in ps.

The front-end electronics integrate the current from each of the 64 channels, and the software calculates the total count $(q)$ :

$$
\mathrm{q}=\mathrm{ka} \int_{-\infty}^{+\infty} \mathrm{t} \Phi(\mathrm{t}) \mathrm{dt}
$$

The definition of the centre of gravity of $\Phi(t)$ is given by the expression:

$$
\mathrm{G}=\frac{\int \mathrm{t} \Phi(\mathrm{t}) \mathrm{dt}}{\int \Phi(\mathrm{t}) \mathrm{dt}} .
$$

Moreover, the integral of the total flux $\Phi(\mathrm{t})$ in the denominator is stable from turn to turn and the total count $q$ gives an expression of the position of the longitudinal centre of gravity.

The sensitivity is given by the slope of the pulse bias and the total count recorded at the medium value of the bias. The useful linear slope is $4 \mathrm{~V} / \mathrm{ns}$ and the standard total count is about 2000 , depending on the vertical $\sigma$, at $4 \mathrm{~V} \mathrm{DC}$. In normal conditions the sensitivity is then two counts per picosecond. That means the total count changes by two counts when the phase changes by 1 ps between the RF signal and the centre of gravity of the bunch. The total count in our detector is done by the sum of all the flash ADCs. These 